\title{
Energy Efficient Protocols for Wireless Sensor Networks: A Survey and Approach
}

\author{
B. P. S. Sahoo \\ School of Math-Stat-Comp Sc \\ Utkal University \\ Bhubaneswar, India
}

\author{
Satyajit Rath \\ CN\&eM Division \\ CSIR - IMMT \\ Bhubaneswar, India
}

\author{
Deepak Puthal \\ Dept. of Computer Sc. \& Eng. \\ NIT Rourkela \\ Rourkela, India
}

\begin{abstract}
Wireless Sensor Networks have limited resources with traditional data gathering techniques. One of the limitations of wireless sensor nodes is its inherent limited energy resource. Therefore, designing an effective wireless sensor network to maximizing the lifetime of sensor node in order to minimize maintenance and maximize overall system performance becomes important. In this article, we have outlined the design factors and challenges in sensor networks. Then, we describe several MAC layer and routing layer protocols proposed for sensor networks. In this paper, we propose an adaptive approach to find an optimal routing path from source to sink when the sensor nodes are deployed randomly deployed in a restricted service area with single sink. Our analysis says our approach reduces the message communication to find a optimal routing path. Hence, the network consumes less energy and increases the lifetime of the network.
\end{abstract}

\section{General Terms}

Wireless Sensor Network, Communication Protocol.

\section{Keywords}

Wireless sensor network, communication protocols, energy efficiency;

\section{INTRODUCTION}

A sensor node in a Wireless Sensor Networks (WSNs)[1-3] is typically equipped with a transducer, a radio transceiver, small micro-controller and a power source (usually batteries) deployed in phenomenon intended to monitor at diverse locations. Sensor nodes are capable of sensing many types of information from the environment including temperature, light, humidity, pressure, wind direction and etc. They usually transmit the acquired data through RF channel to the base station or gateway. Now a days, WSNs has a wide range of application areas such as: industrial process monitoring \& control, robot control, environmental monitoring, habitat monitoring, health care applications, home automation, object tracking, traffic control and etc. However, WSN has its own design and resource constraints issues in practices. Resource constraints include a limited energy, low range communication, low bandwidth, and limited processing power $\&$ storage capacity. The recent research in WSNs intend to convene these constraints by introducing new design concepts, creating or improving existing protocols, building new applications, and developing new algorithms. Many researchers are currently engaged in developing schemes that accomplish these requirements.

In WSNs energy consumption is one of major issues which needs to be carefully consume by sensor node to maximize the network lifetime. Typically the sensor nodes are powered by small batteries which are incapable to power a long period. Generally, the sensors nodes are deployed in a left unattended area. In such situation feeding energy to the battery or replacing batteries is difficult or even not possible too. Therefore, prolonging the network lifetime is an important optimization goal in this aspect. Hence the energy consumption by whole network in every aspect needs to be minimized. The secret to reduce energy consumption lies in power aware designing of each layer of the system and message transmission for data packet. Energy in the sensor nodes fulfills a very important need for real-time data recording from the sensors. So the current research directed towards; how to design an efficient and energy-awareness protocols in order to extend the lifetime of the intact networks.

The amount of energy the network spends for transmitting one data packet can be minimizes by applying data compression [19] techniques, because if the data packet will be heavier it will consume more energy. There are many energy efficient protocol in each layer have been proposed to prevent the energy consumption in sensor nodes too. Moreover, as the sensor nodes are densely deployed in a phenomenon to be monitored, sometimes multiple nodes may sensed the same event and transmit the redundant readings. So in this context, the non redundant data should be transmitted. So in such scenario we an efficient scheme should be chosen which can address it.

Motivated by the above study, in this paper we propose an efficient way to find an optimal routing path for the communication from source node to sink node. Our analysis says it reduces the message communication to find a routing path. Hence it consumes less energy and increases the lifetime of the network.

The rest of the paper is presented as follows. Section II describes the issues of design factors. Few energy efficient MAC layer and routing layer protocol has been reviewed in Section III and IV respectively. Section V contains the details principle of operation of our proposed model. The paper is concluded in Section VI.

\section{DESIGN FACTORS \& CHALLENGES IN WSNs}

The efficient and robust realizations of WSNs are challenging and algorithmic task, because of the unique characteristics and sever limitations of these devices. Sensor network requires the efficient and robust distributed protocols and algorithms with properties such as[3]: (a) Scalability:- Able to operate in extremely large networks composed of huge numbers nodes (b) Efficiency:- Efficient with respect to both energy and time (c) Fault Tolerance:- Network should be able to operate despite of any failure of any nodes.

One of the most crucial goals in designing efficient protocols for WSNs is minimizing the energy consumption. This goal has various aspects [3]: (a) minimizing the total energy spent 
in the network (b) minimizing the number of data transmission (c) combining energy efficiency and faulttolerance by allowing redundant data transmission which, however, should be optimized to not spend too much energy (d) maximizing the number of 'alive' particulars overtime, thus prolonging the systems lifetime (e) balancing the energy dissipation among the sensors in the network.

In this study, we have discussed different MAC protocols and routing protocols proposed for sensor networks from the literature. We described briefly the essential behaviors, advantages and limitations of the protocols wherever possible. More precisely, in this study we have much concentrated on the energy efficiency parameter of the proposed protocols. This section helps the young researcher to find a few approaches which needs to be taken care while designing an energy efficient protocol.

\section{MEDIUM ACCESS CONTROL PROTOCOLS IN WSNS}

One indicated approach to adaptively adjust the transmission power to an appropriate level for generating signal strength, just enough to reach the next hop destination, is to control the power consumption rate of a sensor node and thus to reduce the collision probability [4]. There are various potential energy waste issues in sensor network which needs to be taken care in MAC layers protocols. In MAC layer, the Major sources of energy waste in wireless sensor networks like collision, overhearing, control-packet overhead, idle listening and over emitting should be minimize to prevent the energy wastes. A good MAC protocol should gracefully accommodate changes in network size, node density, and topology. Wireless sensor MAC protocols are broadly classified into two categories: Contention based and Schedule based. The collisions, overhearing and idle listening can be avoid by scheduling transmit $\backslash \&$ listen periods but have strict time synchronization requirements. The contention based protocols on the other hand relax time synchronization requirements and can easily adjust to the topology changes as some new nodes may join and others may die few years after deployment. A survey of MAC protocols for wireless sensor networks has been reported in [6]. The authors in [6], has discussed the protocol's strengths and weaknesses, and also pointed out open research issues with regard to MAC layer design. Many MAC layers protocols have been proposed to prevent the energy in sensor network such as: IEEE 802.11, SMAC [7], AC-MAC, T-MAC, B-MAC, AC-AMC, TRAMA, etc. In our study we will also discuss some few other energy efficient protocol in the rest of this section.

\subsection{Sensor MAC}

Sensor-MAC (S-MAC) [7] is a contention-based protocol which works on the principle of IEEE 802.11. The performance in terms of energy efficiency is better than IEEE 802.11. This protocol prevent energy consumption from four sources of energy wastage like idle listening, collision, control packet overhead and overhearing. Basically these protocols save energy by transition of node periodically with listen and sleep mode. The authors of [8] suggests an adaptive MAC protocol based on S-MAC, named AC-MAC. Combining SMAC and AC-MAC with Dynamic Power Management mechanism [10] suggests a new protocol called ACMAC/DPM. In the mechanism of S-MAC, a node goes to sleep period when they have no data to send or receive. Nodes remain in the listen state and exchange data if they are involved in communication. S-MAC is a well known MAC protocol for energy efficiency in sensor network. The experiment result shows that, on a source node, an 802.11-like MAC consumes 2 to 6 times more energy than S-MAC for traffic load with messages sent every 1-10s [7]. This mechanism is easy to implement and the energy waste reduced by sleep schedules. However the overall efficiency decreases due to the predetermined sleep and listening duration. It is also prone to collision in broadcasting as it does not use RTS/CTS packets. The latency is increased due to the periodic sleep of each node.

\subsection{Real Time MAC}

Sahoo and Baronia have proposed a real-time MAC protocol called RTMAC [20] based on TDMA protocol to address the issue of higher latency in S-MAC protocol with low energy consumption. This protocol conserves energy when a node may not be transmitting or receiving packets by putting it into sleep state. This sleep and wake up pattern saves energy for the sensor node. The author claims that the RTMAC is suitable for real time applications like detection of radioactive radiation, earthquake. The performance of RTMAC protocol has been compared with IEEE 802.11 and S-MAC, and it has been shown that the RTMAC protocol's performance lies in between the two said two protocols in terms of energy efficiency. The basic principle of this protocol is: the sensing area is divided into rectangular grids with ring frames. In each ring frame some number of sensor node has deployed. Lets us assume the sensing area has three rings frames such as: $C_{1}, C_{2}$ and $C_{3}$. For example [20], when $C_{3}$ is transmitting to $C_{2}, C_{2}$ will be in receiving mode and hence should not be transmitting anything to $C_{l}$. Hence, $C_{l}$ (which is the receiver ring of $C_{2}$ ) should not be in receiving mode during that time. Therefore, nodes in $C_{l}$ can sleep when $C_{3}$ is transmitting. This sleep pattern enables RTMAC to save, on an average, 1/3 energy as compared to a conventional TDMA system.

\subsection{Adaptive MAC Based on Dynamic Power Management}

The adaptive MAC protocol for Wireless Sensor Networks, AC-MAC/DPM [10] is a scheduled based protocol which has been designed to address the problem of AC-MAC protocol i.e. frequent transceiver state switches which can lead to the increasing consumption of energy. It is a modified scheme of AC-MAC protocol. This new protocol scheme introduced the Dynamic Power Management (DPM) mechanism into ACMAC to reduce the energy consumption due to the transceiver state switches between idle and sleep. AC-MAC calculates the number of new duty cycles according to sensors traffic load, and uses the number of packets queued at the MAC layer as an indication of the traffic load. Considering $\mathrm{Ni}$ be the number of packets queued at MAC layer for node $i$ and $R_{i}$ be the weighted value, $R_{i}$ can be expressed as [10]:

$$
R_{i}=f\left(N_{i}\right)
$$

Where $f(-)$ is an application-specific function which expresses the desire of a node for transmission. The upper limit of $R i$ can be denoted as [10]:

$$
R i=\frac{T_{\text {frame }}-T_{\text {SYNC }}}{T_{R T S / C T S}+T_{\text {data }}}=\frac{T_{R T S / C T S}-T_{\text {sleep }}}{T_{R T S / C T S}+T_{\text {data }}}
$$

Where Tframe is the duty cycle interval, Tlisten is the listen interval, Tsleep is the sleep interval, TSY NC is the SYNC interval, and TRTS=CTS is the RTS/CTS interval and Tdata is the maximum time for data transmission. 
The basic principle of AC-MAC/DPM is to control the value of $\mathrm{Ti}$ i.e. the sleep time in a reasonable range. The author claims that by redefining the upper limit of Ri, Rimax, the potential energy consumed by state switches can be reduced. In ACMAC/DPM, the sleep time of the reduced duty cycle is equal to the summation of the maximum time for data transmission and the time for transition of state. At the beginning of each basic duty cycle, a node firstly checks the number of packets queued at MAC layer for node $i$, namely $\mathrm{Ni}$, then $\mathrm{Ni}$ is mapped into a right value, $\mathrm{Ri}$. If $\mathrm{Ri}<\mathrm{Rimax}$, the node gets Ri chances of communication within one basic duty cycle, otherwise, the node should be always awake in one basic duty cycle. This approach guarantees low delay or high throughput and reduces the potential energy consumption when the traffic load is high. The simulation result shows the delay of AC-MAC/DPM is lower than the S-MAC protocol.

\subsection{Sift: An Event-Driven MAC Protocol}

The Sift [11] protocol exploits the event driven nature of the sensor networks for MAC protocol design. It is a contention window based MAC protocol. This protocol adopts a typical random access protocol such as CSMA or CSMA/CA and uses a fixed size contention window with a non-uniform probability distribution for choosing the contention slot for a node. If no node starts to transmit in the first slot of the window, then each node increases its transmission probability exponentially for the next slot, assuming that the number of competing nodes is small. The main motivation of the Sift protocol is address the spatially-correlated contention issue in sensor network. More precisely, it address the issue of when multiple nodes in the same neighborhood all sense an event they need to transmit information about and a subset of the nodes that observe the same event report it. This protocol is designed on the premise that often only a subset of all the nodes need to send their reports and suppress the rest. This protocol achieved very low latency, which is an important parameter in sensor network. The simulation shows the Sift performs better and surpasses 802.11 for 8 or more number of flows. However, one of the main issues in Sift, that it does not focus on the energy consumption issue since it constantly listens during the backoff period like 802.11. However, it is possible to integrate the Sift mechanisms with other wireless MAC protocols that focus on minimizing the energy consumption.

\section{ROUTING PROTOCOLS IN WSNS}

Routing in WSNs is very challenging due to the inherent characteristics that distinguish the wireless sensor networks from other wireless networks like mobile ad hoc networks or cellular networks [4]. The sensor network adopts two basic schemes for energy savings in network layer such as[3]: Power-Aware Routing and Maximum Lifetime Routing. The main aim at the network layer is to find the route to transmit data from sensor nodes to the sink in an energy-efficient and reliable manner in order to maximally extend the lifetime of the network. The design of routing protocols in WSNs is influenced by several distinguishing characteristics related to system architectural model which needs to be keeping in mind while designing a network layer protocol for sensor network. WSNs divide routing protocol into three categories such as: Flat, Hierarchical, Location-based. The distinction of these types is described in [4] as: in flat-based routing, all nodes are typically assigned equal roles or functionality, in hierarchicalbased routing, nodes will play different roles in the network and in location-based routing, sensor nodes' positions are exploited to route data in the network. Many routing protocols have been proposed in wireless sensor network to address the energy issue in sensor node such as: LEACH, LEACH-C and PEGASIS. We will discuss few more energy efficient protocol in the rest of this section.

\subsection{Sensor Protocols for Information via Negotiation (SPIN)}

Kulik et.al. in $[14,15]$ proposed an adaptive protocols called Sensor Protocols for Information via Negotiation (SPIN) that disseminate all the information from each node to every node in the sensor network. This enables a user to query any node and get the required information immediately. These protocols make use of the property that all sensor nodes have same data in the network that other nodes do not possess. The SPIN family of protocols uses data negotiation and resourceadaptive algorithms. SPIN protocol delivers $60 \%$ more data with consuming same amount of energy as flooding [9]. Nodes running SPIN perform meta-data negotiations before any data is transmitted. Meta-data describes about the data but the size is very less than the actual data. This assures that there is no redundant data sent throughout the network. For example, sensor nodes use their unique ID to report metadata. In addition, SPIN has access to the current energy level of the node and it runs based on how much energy is remaining. These protocols work in distributed environment, when a user does not request any data. The SPIN family is designed to address the deficiencies of classic flooding and gossiping by negotiation based data transmission. The SPIN family of protocols is designed based on two basic ideas; one is sensor node operates more efficiently and conserve less energy by sending the meta data, other one is to reduce the implosion, bandwidth consumptions, duplicate messages sent to the same node and save energy by introducing the negotiation based data transmissions.

The SPIN family of protocols includes two different protocols are called SPIN-1 and SPIN-2, which introduced negotiation before transmitting data in order to ensure that only useful information for other node will be The SPIN family of protocols includes two different protocols are called SPIN-1 and SPIN-2, which introduced negotiation before transmitting data in order to ensure that only useful information for other node will be transferred. The SPIN-1 protocol is a 3-stage protocol. It uses three types of packet called ADV, REQ and DATA packets. This is the on-demand types of protocol, when one node has some data to share among its neighbours first it sends an ADV packet to all of its neighbours. Those nodes show their interest to take the data from their neighbour it returns back with an ACK packet. The sensor node sends the DATA packet to those nodes from where it receives the ACK. This is the three stage process through which nodes share their data in the networks. It can also follow the same process in-order to deliver the data towards the sink node. An extension to SPIN-1 is SPIN-2, which incorporates thresholdbased resource awareness mechanism in addition to 
negotiation. When energy level of the node is enough, SPIN-2 works like 3-stage protocol of SPIN-1. However, when the energy level goes down node reduces its participation in the protocol, i.e., it participates only when it has enough energy that it can complete all the three stages of the protocol without going below the low-energy threshold. SPIN protocols efficiently disseminate data, while maintaining no perneighbour state. These protocols are well-suited for an environment where the sensors are mobile because they base their forwarding decisions on local neighbourhood information. Other protocols of the SPIN family are:

- SPIN-PP: This protocol is designed for a point to point communication.

- SPIN-EC: This protocol works similar to SPIN-PP, but with an energy constraints added to it.

- SPIN-BC: This protocol is designed for broadcast channels.

- SPIN-RL: When a channel is lossy, a protocol called SPIN-RL is used where adjustments are added to the SPIN-PP protocol to account for the lossy channel.

In [9] it also proved that SPIN protocol work efficiently to deliver the critical data towards the sink node in the mobile sink sensor network. In which network all the sensors are static other than the sink node. Sink moves randomly in the network to collect the data from the static sensors. SPIN protocol implement only when the sensor have critical data to send and sink not in its range.

\subsection{Geographical Routing}

Seada et.al. in [17] described geographical routing uses a greedy forwarding approach to forward the packet from the source to the destination. Sensor node chose the neighbors which are closed to destination and forward the data packets to the corresponding neighbors. Geographical routing assumes that the network is deployed densely; nodes know their own location and their neighbor's locations. There are several novel forwarding strategies are proposed to improve the performance of geographic routing. These forwarding strategies can be divided into two categories: distance-based and reception-based. In distance-based forwarding, a node only knows the distance of its neighbors and in receptionbased forwarding the packet reception rates of its neighbors are also known. Distance-based forwarding approach follows the original greedy forwarding and distanced-based blacklisting. In greedy forwarding approach, each node forwards packets to the neighbor closest to the destination based on a minimum response rate and selects neighbors with highest distance, independent of the response rate. In distancebased blacklisting, each node blacklists neighbors that are more than a certain distance threshold from itself. The blacklist distance threshold is set a nominal radio range. Packets are forwarded to those neighbors that are closest to the destination and distance is less than the threshold from the current forwarding node.

\subsection{Rumor Routing}

Braginsky et.al. in [16] described rumor routing is a variation of directed diffusion and is mainly proposed where geographic routing is not feasible. Generally, directed diffusion floods to spread the query in the network when there is no geographic condition to diffuse tasks. Sometimes flooding is unnecessary because there is very little amount of data requests from the sensor nodes. And flooding is required when number of event is less and number of query is very large. The key idea is to route the queries to the nodes that have observed a particular event rather than flooding the entire network to retrieve information about the occurring events. In order to flood events through the network, the rumor routing algorithm employs long-lived packets, called agents. When a node detects an event, it adds such event to its own local table, called events table, and generates an agent. Agent travels the network in order to propagate information throughout the network. When a node generates a query for an event, the nodes responds the query about the rout after checking from the event table. As, there is not flooding the whole network, which reduces the communication cost. Rumor routing maintains only single path between source sensor node and sink which is opposite to directed diffusion where data routs through multiple paths. Rumor routing achieves energy savings, when compared with flooding and can also handle node's failure results showed by simulation. Rumor routing performs well when the numbers of events are small. When there are large numbers of events, the cost of maintaining agents and event-tables at each node becomes infeasible. Rumor routing is controlled by different parameters used in the algorithm such as time-to-live (TTL) concerning to queries and agents. Since the nodes are aware of events through the event agents, next hop selection in rumor routing is defining the rout of an event agent.

\subsection{SPEAR: Sensor Protocol for Energy Aware Routing}

Bhuvaneswari et. al. in [18] proposed a similar clustering scheme as LEACH protocol. In their proposed model they designed the cluster head election process, which made energy aware leading in case of heterogeneous node. In LEACH pro-tocol it selects the cluster head randomly to extend the network life time. However it does not scale the network life time well in a heterogeneous condition due to the random cluster head election scheme. Further the protocol also ensures a uniform distribution of cluster heads in the service area and cluster head election is based on a threshold distance. In LEACH protocol it selects cluster head by stochastic process to which leads $\mathrm{CH}$ may not be uniform distributed area. The protocol maintains a minimum threshold distance between any two cluster head nodes leading to a uniform energy load distribution among the nodes and network model assumptions made are same as in LEACH protocol architecture. The SPEAR protocol is operated in two phases like setup phase and steady state phase. 


\section{PRINCIPLE OF OPERATION OF OUR PROPOSED MODEL}

The unknown number of nodes has been randomly deployed in a restricted service area with single sink within a overlapping radio range of its neighbor nodes. Each sensor node in the service area maintains a routing table [13] for them. Utilizing this routing table each node finds a path to the sink node. As the wireless sensor network is deployed in an unattended environment, so the major challenges to maximize the network life time by minimizing the communication cost. We have proposed an adaptive approach which can be achievable by finding the optimal routing path with unicast the data packets from source node to the sink.

\subsection{Routing Table Construction}

In this section, we discuss the construction of the routing table in detail. We have represented the routing table as a matrix of $\mathrm{n} \times \mathrm{n}$. Where $\mathrm{n}$ is the total number of nodes in the network. The whole network is represented as a rooted tree structure as shown in figure 1 . The tree is decoded into the matrix by applying our proposed algorithm. The row number of the matrix represents the node (parent node) while column number represents children of parent node, The intersection between row and column gives a cell representing the link between parent and child. If there is a network connection between the two nodes, then the value of the cell will be 1 , otherwise 0 . Each row that has 1 's is a parent node, leaf nodes are rows without 1 's and disconnected node is a node without 1 's in rows and columns. Root node is a row that has one along the diagonal of the matrix. Now we explain the basic idea of the construction of the routing table. As the sensor nodes are deployed randomly with overlapping radio range, one sensor node can fall within the radio range of many nodes and it can communicate with those sensor nodes. But we have restricted the communication to only one node with one hop distance, i.e. only to its parent node. In other word the node can only communicate with its parent even it practically can communicate with the nodes which are in the radio range of its own. In our routing table one particular node only store its next hop address towards the sink. When at any moment the node receives a packet from its child it simply transfers that packet to its next-hop. In this way an energy efficient optimal path can be found from the source to sink node with minimal hop and unicast the packets. This optimal path is used as the routing path from the source to sink. In meanwhile the routing table is updated dynamically based on the link status of the node within the network. This gets triggered when any of the data packets could not get delivered to its next hop. This is in dead, increase the chances of failure for the data packets in reaching to the sink node. Our algorithm follows the steps below to set up links in the routing table with some assumptions. Assumption 1: Network contains single sink with random numbers of sensor node. Assumption 2: Signal strength between any two nodes is same.We construct the routing table based on our above proposed algorithm. Each node maintains a routing information table to find an energy-efficient routing path to the sink. We will discuss in the next section, how the data packets is being sent from the source to sink for with a typical example.
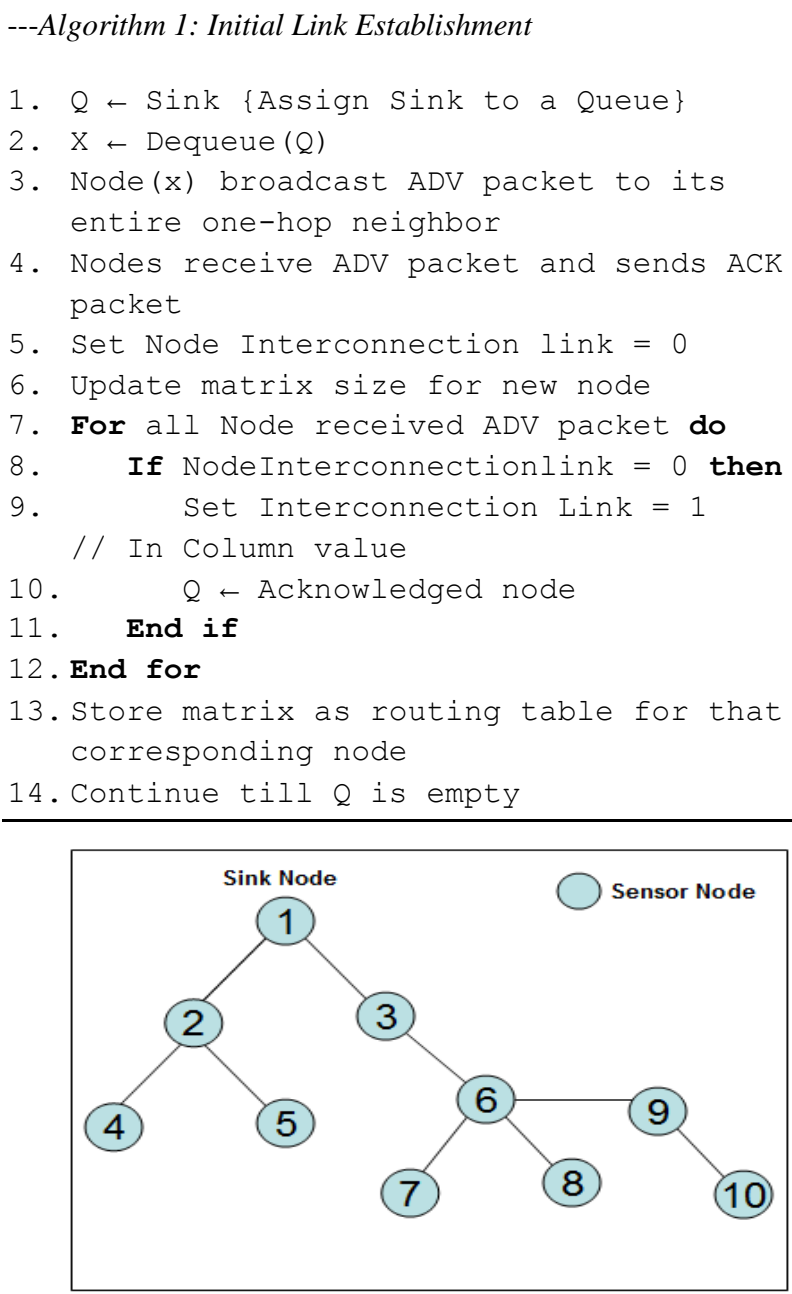

Figure 1: Sensor node deployment in filed.

\begin{tabular}{|l|l|l|l|l|l|l|l|l|l|l|}
\hline & 1 & 2 & 3 & 4 & 5 & 6 & 7 & 8 & 9 & 10 \\
\hline 1 & 1 & 1 & 1 & 0 & 0 & 0 & 0 & 0 & 0 & 0 \\
\hline 2 & 0 & 0 & 0 & 1 & 1 & 0 & 0 & 0 & 0 & 0 \\
\hline 3 & 0 & 0 & 0 & 0 & 0 & 1 & 0 & 0 & 0 & 0 \\
\hline 4 & 0 & 0 & 0 & 0 & 0 & 0 & 0 & 0 & 0 & 0 \\
\hline 5 & 0 & 0 & 0 & 0 & 0 & 0 & 0 & 0 & 0 & 0 \\
\hline 6 & 0 & 0 & 0 & 0 & 0 & 0 & 1 & 1 & 1 & 0 \\
\hline 7 & 0 & 0 & 0 & 0 & 0 & 0 & 0 & 0 & 0 & 0 \\
\hline 8 & 0 & 0 & 0 & 0 & 0 & 0 & 0 & 0 & 0 & 0 \\
\hline 9 & 0 & 0 & 0 & 0 & 0 & 0 & 0 & 0 & 0 & 1 \\
\hline 10 & 0 & 0 & 0 & 0 & 0 & 0 & 0 & 0 & 0 & 0 \\
\hline
\end{tabular}

Figure 2: Routing table presented as matrix.

\subsection{Optimal Path Finding from Routing Table}

In this section we explained the problem for finding the optimal path from source to sink from routing information matrix. We find the routing path for each source node in the WSNs from the constructed routing table in a straight forward manner as state in the algorithm 2. Therefore, when any source node transmits data to the sink, only those nodes who 
are in the routing path are active, and other nodes continue to sleep. In figure 3 , suppose a routing path considered as $8 \rightarrow 6$ $\rightarrow 3 \rightarrow 1$ at a moment. Initially all nodes keep sleep state, source node 8 start sending data to the sink at any time when triggered by an event of interest. The circle in figure 3 shows that the node 6,7 and 9 are in the radio range of node 8 . When node 8 sends data to sink, all the nodes that fall under radio range of 8 should get this data packet. As we have restricted the communication with unicast only, the node 8 sends the data packet to its parent or next-hop, i.e. node 6 . We have proposed the algorithm 2, to find the optimal path from source to sink which steps as below. Our adaptive approach minimizes the unnecessary flooding or broadcasting of data packets as we have adopted the unicast technique to transfer. After this operation if there are data to send/receive, they keep awake, otherwise they switch sleep state.

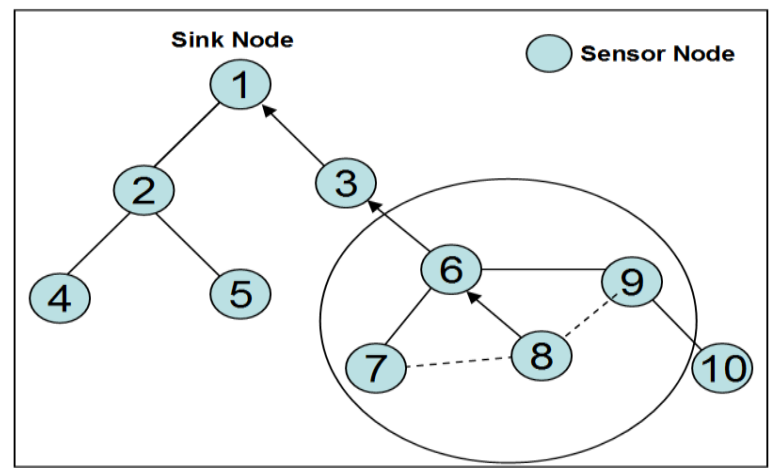

Figure 3: Deployment filed with radio range of a node.

$--$

Algorithm 2: Routing Path from Source to Sink

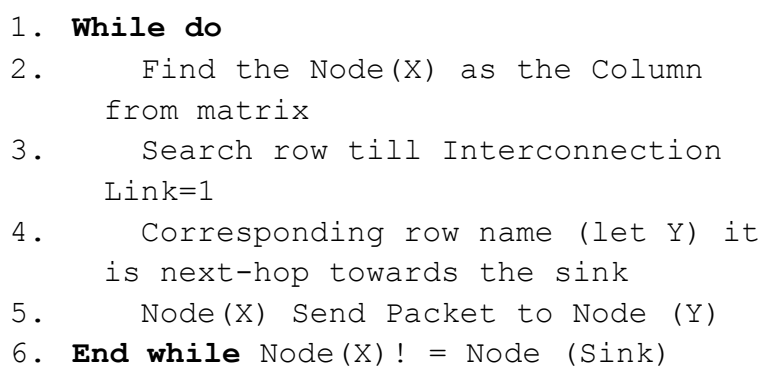$$
--
$$

\section{CONCLUSION AND FUTURE WORK}

In this paper, we have studied several energy efficient MAC layer and routing layer protocols for wireless sensor networks and deeply analyzed the problem for finding a optimal routing path from the source node to sink. Subsequently, we have proposed an adaptive approach for finding the optimal routing path with unicast of data packets to minimize the message transmission which concludes with the energy efficiency of the network and maximizes the network lifetime. Our future work entails developing and testing our model in a simulated environment.

\section{REFERENCES}

[1] Ian F. Akyildiz, Weilian Su, Yogesh Sankarasubramaniam, and Erdal Cayirci, "A Survey on Sensor Networks," in IEEE Communications Magazine, August 2002.

[2] I.F. Akyildiz and W.Su and Y. Sankarsubramaniam, "Wireless sensor networks: a survey, in IEEE Communications Magazine,, August 2004.

[3] Sotiris Nikoletseas, "Algorith for Wireless Sensor Networks: Design, Analysis and Experimental Evolution", in: the Proceedings of 5th International Workshop, WEA 2006, Cala Galdana, Menorce, Spain, May 2006, Springer.

[4] Nikolaos A. Pantazis, Dimitrios D. Vergados, "A Survey on Power Control Issues in Wireless Sensor Networks," in IEEE Communication Surveys, The Electronic Magazine of Original Peer-Reviewed Survey Articles, $4^{\text {th }}$ Quater, Volume 9, No. 4, 2007.

[5] Jamal N. Al-Karaki, Ahmed E. Kamal, "Routing Techniques in Wireless Sensor Networks: A Survey," in IEEE Wireless Communications, December 2004.

[6] Ilker Demirkol, Cem Ersoy, and Fatih Alagz, "MAC Protocols for Wireless Sensor Networks: A Survey," in IEEE Communications Magazine, April 2006.

[7] Wei Ye, John Heidemann, Deborah Estrin, "An EnergyEfficient MAC Protocol for Wireless Sensor Networks, in IEEE INFOCOM, 2002

[8] Jing Ai, Jingfei Kong, Damla Turgut, “An Adaptive Coordinated Medium Access Control for Wireless Sensor Networks, in IEEE, 2002

[9] Deepak puthal and Bibhudatta Sahoo "Adaptive Protocol for Critical Data Transmission of Mobile Sink Wireless Sensor Networks", in proceeding of IEEE International Conference on Computing, Communication and Applications (ICCCA-2012) Dindigul, India, Feb. 2224, 2012.

[10] Feng Li, Yun Li, Weiliang Zhao, Qianbin Chen, Weiwen Tang, "An Adaptive Coordinated MAC Protocol Based on Dynamic Power Management for Wireless Sensor Networks," in IWCMC06, ACM, Canada, 2006.

[11] K. Jamieson, H. Balakrishnan, and Y. C. Tay, "Sift: A MAC Protocol for Event-Driven Wireless Sensor Networks," MIT Lab. Comp. Sci., Tech. rep. 894, May 2003, available

at
http://www.lcs.mit.edu/publications/pubs/pdf/MIT-LCSTR-894.pdf

[12] Kemal Akkaya, Mohamed Younis, “A survey on routing protocols for wireless sensor networks, Ad Hoc Networks, 3 (2005) 325349, Elsevier, 2003.

[13] Shujuan Liu, Yuebin Bai, Mo Sha, Qingyuan Deng, Depei Qian, "CLEEP: A Novel Cross-Layer EnergyEfficient Protocol for Wireless Sensor Networks, IEEE 2008.

[14] J. Kulik, W. R. Heinzelman, and H. Balak- rishnan, "Negotiation-based protocols for disseminating information in wireless sensor networks", Wireless Networks, Volume: 8, pp. 169-185, 2002.

[15] W. Heinzelman, J. Kulik, and H. Balakrishnan, "Adaptive Protocols for Information Dissemination in Wireless Sensor Networks" in: Proc. of $5^{\text {th }}$ ACM/IEEE 
Mobicom Conference (MobiCom '99), Seattle, WA, August, 1999. pp. 174-85.

[16] D. Braginsky and D. Estrin, "Rumor Routing Algorithm for Sensor Networks," in: Proceedings of the First Workshop on Sensor Networks and Applications (WSNA), Atlanta, GA, October 2002.

[17] K. Seada, M. Zuniga, A. Helmy, B. Krishnamachari, "Energy-efcient forwarding strategies for geographic routing in lossy wireless sensor networks", in: Proceedings of the Sensys04, Baltimore, MD, 2004.

[18] Bhuvaneswari.P.T.V, Vaidehi.V and Shanmugavel.S, "SPEAR: Sensor Protocol for Energy Aware Routing in
Wireless Sensor Network“, in: WCSN-2007, pp. 74-78, Dec. 13-15, 2007.

[19] Tommy Sazalapski and Sanjay Madria, "Energy Efficient Real-Time Data Compression in Wireless Sensor Network", in: 12th IEEE Inc. Conf. on Mobile Data Management, IEEE Computer Society, 2011

[20] Anirudh Sahoo and Prashant Baronia, "An Energy Efficient MAC in Wireless Sensor Networks to Provide Delay Guarantee," in Proceedings of the 2007 15th IEEE Workshop on Local and Metropolitan Area Networks, 2007. 